\title{
An Intelligent Hospital Device for Health Caring Assistance of Patients with Motor Disabilities
}

\author{
Eduardo Vázquez-Santacruz, Mariano Gamboa-Zúñiga \\ CGSTIC, Cinvestav-IPN, Mexico, D.F., Mexico \\ \{efvazquez, mgamboaz\}@cinvestav.mx \\ http://administracion.cinvestav.mx/SecretariadePlaneacion/ \\ CoordinacindeServiciosdeApoyo/CGSTIC.aspx
}

\begin{abstract}
In this document we present an intelligence device which includes a monitor system for automatic movements of a robotic hospital bed based on posture classification and identification. This functionality has been developed in response to the requirements defined by the application of a diagnostic methodology [10]. Our diagnostic methodology applied on a Mexican public hospital allowed us to understand that the problem of mobility in elderly people and disabled people involves solutions like devices that attend these individuals to achieve movement without many complications. The movement of these patients can be performed routinely or sporadically during their stay in hospital. For patients who require certain routine application of this movement is designed an intelligent monitor system. This intelligent system allows medical experts to program the movements of the robotic bed considering the patient's posture and time. We will show a methodology for bodies classification in real time. Our work considers the problem of human posture classification with few information (sensors), applying digital process to expand the original data of the sensors and so get more significant data for the classification, however, this is done with low-cost algorithms to ensure the real-time execution.
\end{abstract}

Keywords: intelligent hospital devices, intelligent monitor, assistive devices, health care technology, applied artificial intelligence, posture recognition.

\section{Introduction}

In recent years the artificial intelligent systems (IS) have been used in several applications such as industrial control, robot control, traffic surveillance, remote sensing, and speech recognition to mention a few. In particular, IS applied to medical environments has been a challenging task due to the high risk decisions in the diagnosis, monitoring and care of patients. However, in rehabilitation of patients with limited or restricted mobility, as is the case of geriatric patients, IS have been used to control the positioning of robotic hospital beds to prevent the appearance of bed-pressure ulcers, also in activity monitoring and bed-rails 
control $[13,14,15,16]$. Most of the systems for automatic control of hospital bed positions are based in the detection of the posture of patients on the bed using presence sensors, digital cameras, thermal cameras and mattress pressure sensors [19,20,21]. Modern hospital beds are able to adopt twelve positions ranging from the home position to the sit-to-stand position, but do not have a mechanism to prevent accidents when the change of positions takes place. Some of the most common accidents when the bed is moving are the downfall of patients, bad posture when the bed is moving, and injuries caused by improper use of motion controls. In this research we propose an IS in order to reduce the risks of operating a hospital bed with multiple-positions. The main objective of our IS is to prevent accidents when the bed is moving, this is done by detecting the posture of patients using a mattress pressure sensor. We identify the posture of patients by performing an analysis and classification of the pressure distributions using an initial training set of correct postures for all bed positions. The proposed IS can be used to assists the medical staff and also provides a mechanism for an automatic and non-assisted control of bed positioning. Monitoring of leaning people is often used in a variety of hospital

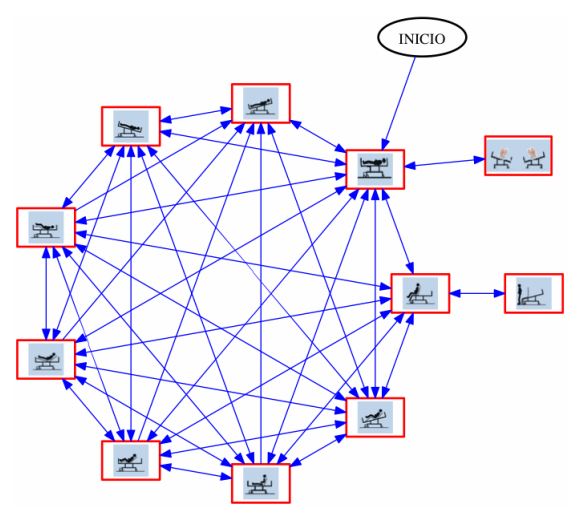

Fig. 1. Transition graph of all possible positions of the robotic hospital bed.

process as geriatrics, rehabilitation, orthopedics and even used in psychology and sleep studies [22]. Although patients monitoring is usually doctors and nurses activity, this can be automated to have better control of the patients in any time [20] doing human intervention only to rate different positions for which patient has passed, the time spent in each posture and incurred transitions. This automatization process is not new, as there are many attempts to recognize the position of a body in a given surface $[20,13,14,16,15]$, most of the systems for posture recognition are based on the detection of the posture of patients on the bed, using presence sensors, digital cameras, thermal cameras or mattress pressure sensors [19,20,21]. Digital and thermal cameras achieve high resolution. However, they are affected by environment illumination, temperature 
conditions and occlusions. Mattress pressure sensors are invariant to illumination and temperature conditions, all the same these sensors have a limited resolution and accuracy. Commercial pressure sensors are available with resolutions from 4 to 1024 sensor units, higher resolution are prohibitively expensive for many applications. Recent developments in the fabrication of low cost pressure matrix sensors are based on organic devices. In [23] a flexible $32 \times 32$ array of pressure sensors have been developed for skin sensitivity with a density of 10 units per inch, it is based on organic field-effect transistors. However, it is limited to small areas and are mostly used in applications such as tactile displays and mobile devices. In [24] pressure sensors based on organic thin film transistors was developed and applied to wearable electronics, textiles and skin for robots. On the other hand, proposed pressure sensors using resistive devices have been tested at highly sensitivity and very short response times that can be inexpensively fabricated over large areas $[25,26]$. Thus, given the high cost in the fabrication of array pressure sensors, is necessary a methodology that use the few data to solve the classification issue. We choose four basic positions according to the famous study by professor Chris Idzikowski [22], who classified the most common sleeping positions in six classes, that is, foetus, log, yearner, soldier, freefaller and starfish. These positions can be grouped in four basic classes: left lateral decubitus position or foetus, right lateral decubitus position of which the log and yearner positions belong to this class, supine position of which the soldier and starfish belong to this class, and prone position or freefaller. We use a flexible array pressure sensor with 448 units distributed in an area of $1860 \times 886 \mathrm{~mm}$. The pressure sensors are based on variable resistive sensors at 4096 pressure levels and normal resistance from 1 to 50 kiloOhm [27]. We construct a database with pressure levels (scaled to 0-255 range) transformed by a normalization process and apply it the HOG algorithm [17], the database contain samples of four postures showed in Fig. 1. With this database, we can construct a classification model using a support vector machine (SVM) classifier [28]. Section 2 explains the intelligent monitor which controls the robotic bed, in section 3 we refer to the image descriptors. Section 4 describes the way in which we make the recognition of the position of patients. Section 5 shows some results and finally Section 6 refers about conclusions.

\section{Intelligence System for Automatic Control of Bed Positions}

A robotic hospital bed is able to adopt several positions depending on the needs for a particular patient, also can be programmed to perform a series of movements in a period of time. We have a transition diagram (Fig. 1) of our robotic hospital bed of the most used positions by medical specialists. The initial position is the home position to which all other positions can reach, with the exception of the sit-to-stand position. The transitions of the robotic bed are performed by means of mechanical actuators, and the time it takes to go from one position to another depends on the weight of the patient and the current 
position. When a transition is performed may happen that the patient falls or can be hurt by being in a bad posture, even when a specialist is operating the robotic bed. The IS will be able to detect if the patient is in a correct position in order to perform the requested transition, and will send a visible alert to prevent possible downfalls. The Fig. 3 shows the main stages of the IS for the posture recognition: in the initial stage the pressure distributions is obtained from the pressure sensor array, in the second and third stages an analysis and pre-processing is performed, also a feature extraction using HOG [17] and SHIFT [18] descriptors are applied over the pressure distributions that are considered as gray scale images; in the fourth stage a database of features is constructed, and in the last two stages we construct a model for feature classification and prediction, we compare the results of three classifiers such as Support Vector Machines, Decision Trees and Bayes-naives Networks. To simplify the posture recognition we consider three basic postures: the right lateral decubitus, supine and the left lateral decubitus positions (see Fig. 2), and since that the prone position is almost the same as the supine position, its detection is achieved by an analysis of the pressure distribution. The Fig. 2 shows the three basic correct positions displayed as gray scale images, obtained from simulated data of the pressure sensor array.

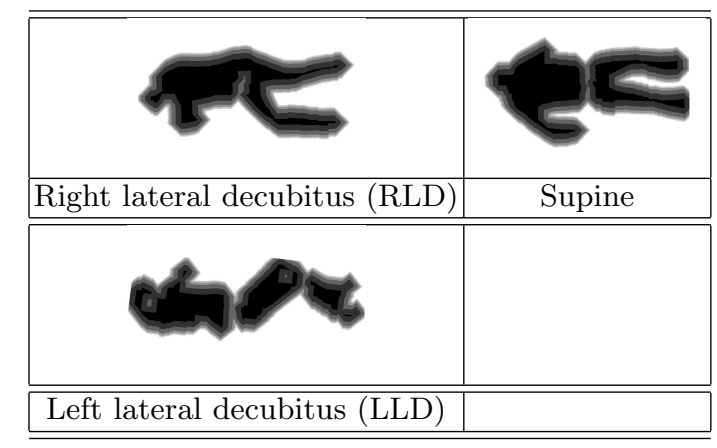

Fig. 2. Three basic correct positions obtained from simulated data.

Thus, the database of features (see Fig. 3) is constructed such as it contains three set of equal size of the three basic positions and an appropriate number of its variants. The database of features is then used to construct the model of the classifier in order to make predictions of pressure distributions that do not belong to the database.

\section{$3 \quad$ Image Descriptors}

Finally, the IS for posture recognition can be used to control the actuators of the robotic hospital bed in a semi-or automatic way, and can prevent accidents when the bed is moving slowly. Thus, when a bad posture is detected then the 


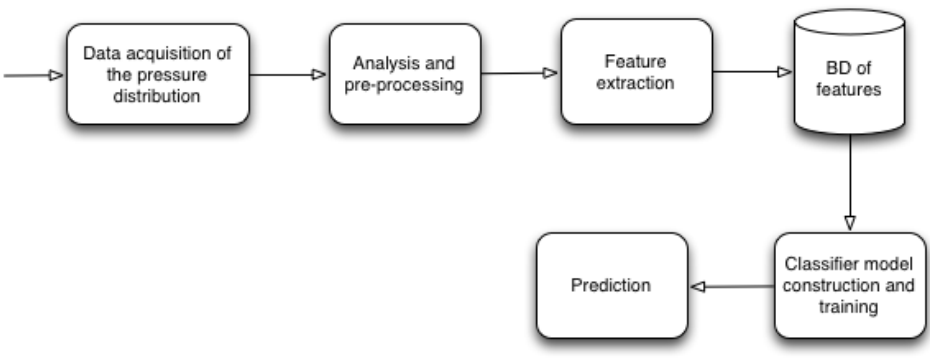

Fig. 3. Main blocks of the IS for posture recognition.

IS send a signal to the actuators either to stop the transition movement or to return to the previous position. A descriptor is a representation of an image, it characterizes the most important features of interest. Descriptors are used in many applications such as image classification and recognition. For these tasks, the desired properties of an image descriptor are the invariance with respect to rotation, scaling, perspective and illumination, several descriptors exist as SIFT [32], SURF [33], DENSE-SIFT [34] and others, but given the features of our image (low quality and gray scale) and mainly due their low computational cost, we decide to use the HOG descriptor, in experiments, HOG showed superior results at runtime compared to SIFT, Dense-SIFT and SURFT; commonly used in posture recognition works [35].

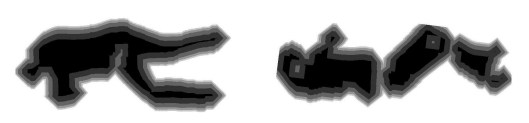

a) LLD (class 1) b) RLD (class 2)
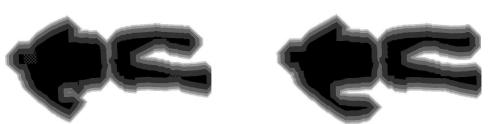

c) Supine (class 3) d) Prone (class 4

Fig. 4. Simulated basic posture positions.

\subsection{HOG descriptor}

HOG (Histogram of Oriented Gradients) descriptor is used in computer vision and image processing for object detection. This descriptor is based on the number of occurrences of the gradient directions in specific parts in an image. The main idea behind the HOG descriptor is that the local shape and appearance can 
be described as the distribution of gradient intensities. Based on that fact, an image is divided in several overlapping blocks, and for each block a histogram of gradient directions is computed. Thus, the HOG descriptor is the composition of all histograms for each block. Fig. 5 shows an example of a simulated pressure image and its gradient directions. The size of the HOG descriptor depends on the size of the blocks and on the number of bins in which the interval from 0 to 180 degrees is divided. We use the HOG descriptor to characterize each pressure image. Therefore, for each pressure map, a vector of gradient directions is constructed. We consider that the histogram of gradient directions can be used for image classification, since that small changes in the pressure images are easily detected as changes in gradient directions.

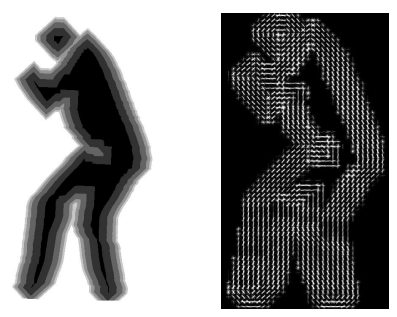

Fig. 5. From left to right: simulated pressure map and image gradient directions.

\section{Methodology for Posture Recognition}

The proposed methodology takes the raw data of sensors and transforms it into HOG image, this representation as HOG image will be used as input in one SVM classifier, the final output is the classifier prediction about the position. Our system receives as input one array of 448 elements, with pressure levels (0-4096 units of pressure), each of which represents one sensor in the surface where a person is lying down, these sensors are distributed in 32 rows and 14 columns. Fig. 6 shows the main blocks of the proposed methodology for posture recognition. In the initial stage, the pressure distribution is obtained from the pressure sensor array, data obtained of the sensors is in range of 0 to 4096 levels of pressure, where 0 is the maximum pressure possible and 4096 is null pressure. Then, in the second block we transform raw data taking three considerations, first, the pressure applied by human body (considering weight between 40 to $150 \mathrm{Kg}$ ) is between 2500 to 4096 in the scale of pressures (see Fig. 7), then we can cut the range only to human body requirements and gain definition. Second consideration is an array escalation from 2500-4096 scale to 0-255 scale for process the array as gray scale image in the next block. The final consideration of the second block is applying a scale algorithm for images to obtain interpolated image of raw pressure data. In third stage, we use a feature extraction using HOG 
descriptor and applying it to the pressure distributions that are considered as gray scale images. In the stage delimited with broken line, we make the SVM model, the first three stages are repeated several times with one human body in different positions (we consider positions described in Fig. 3), and we make a data base with these, after we use these data base for make a SVM model, how we know the position in the which was taken each measurement, we have a pressure interpolated and classified data base, then we can construct a SVM model that predicts the four positions of the Fig. 3. When SVM model is ready, we can use it in order to monitor person movements, the fourth stage implies to take this SVM model and to use it as input the first three stages output, then the fifth stage have an accurate prediction.

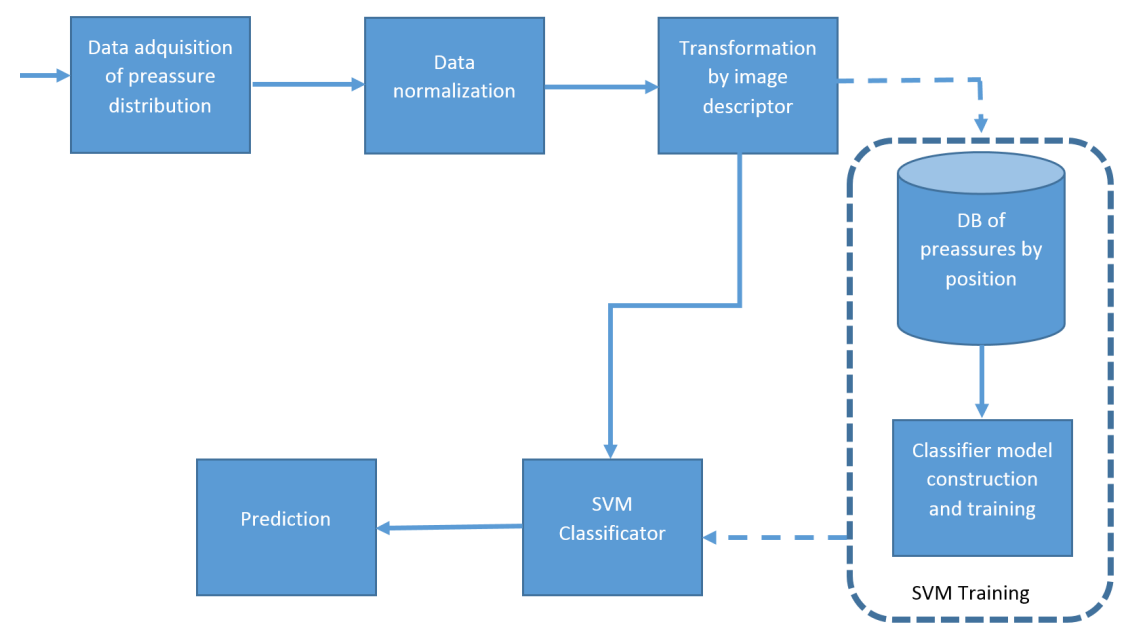

Fig. 6. Main blocks of methodology for posture recognition

\section{$5 \quad$ Results}

\section{$5.1 \quad$ Pressure Maps Database}

The pressure sensors array consists of two independently modules with a resolution of $16 \times 14$ units in an area of $930 \times 886 \mathrm{~mm}$. Each pressure sensor has a response between 0 to $25 \mathrm{~kg} / \mathrm{cm}^{2}$. We develop an interface to synchronize the two modules at a frequency of 100 measurements per second. We construct four different pressure map data set for classification: Each data set consists of four classes, for every posture position (see Fig. 3). For each class we generated 501 instances. We choose this number of instances according to study in [36]. Each data set has a number of 2004 instances and each data set in stored as individual data base, only after of applying the methodology described in section 4 . Fig. 8 


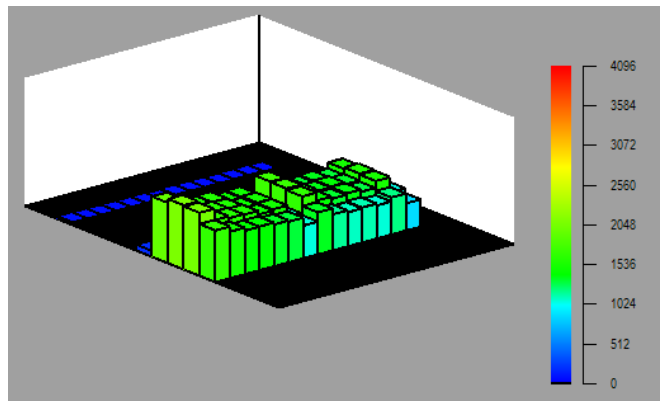

Fig. 7. Pressure levels obtained from posture of a person.

shows some examples of interpolated pressure maps. We normalize the range of values of each attribute vector, this was done in order to improve the performance of the classifier. As we see in Fig. 8 the pressure maps obtained are more intuitive than the showed in Fig. 7.
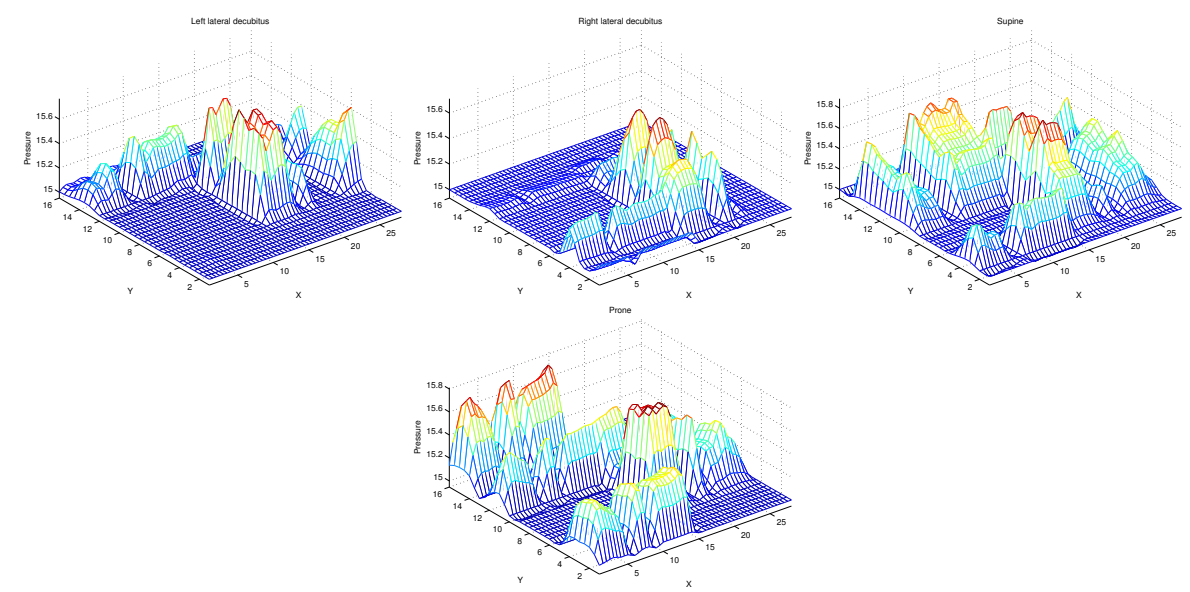

Fig. 8. Interpolated pressure maps corresponding to the four basic posture positions (see Fig. 3) before of apply the proposed methodology.

\subsection{Posture Classification Using Pressure Maps}

We use the LibLINEAR and LibSVM toolbox to perform the posture classification. Table 1 shows the results of the classification using the three databases with the proposed methodology. The classification results were obtained using 10-fold cross-validation. As can be seen the SVM with a linear kernel generates good results using the raw and interpolated data. Using image descriptors, however, 
has a better performance with a RBF kernel. The advantage of using image descriptors is that the time to construct the model of the classifier is slower than using raw and interpolated data. We need not only the percentage of correctly classified instances, for a real-time process we need to verify the computing time, logically the data sets with many elements will take longer time, but we need to found the balance between assertiveness and runtime. Table 2 shows the time that take each dataset for be processed by cross-validation in one computer with processor Intel pentium T4500 $(2.3 \mathrm{GHz})$. Also, it shows results which are obtained by using cross-validation algorithm that process 35,000 sensor arrays inputs, then in this table we can show the number of sensor arrays what can be processed per second. Taking the results of tables 1 and 2, we can see

Tabla 1. Percentage of correctly classified instances.

\begin{tabular}{|l|c|c|}
\hline & Linear-SVM & RBF-SVM \\
\hline \hline $640 \times 640$ & $51.05 \%$ & $54.10 \%$ \\
$320 \times 320$ & $96.21 \%$ & $98.57 \%$ \\
$100 \times 100$ & $98.54 \%$ & $99.01 \%$ \\
$32 \times 14$ & $84.32 \%$ & $85.89 \%$ \\
\hline
\end{tabular}

Tabla 2. Percentage of correctly classified instances.

\begin{tabular}{|l|c|c|}
\hline & Time $($ seconds) & Arrays per second \\
\hline \hline $640 \times 640$ & 5412 & 6 \\
$320 \times 320$ & 977 & 34 \\
$100 \times 100$ & 72 & 486 \\
$32 \times 14$ & 17 & 2058 \\
\hline
\end{tabular}

that the better performance is obtained by $100 \times 100$ interpolated data set, that has $99.01 \%$ of assertiveness and can process 486 data arrays per second (although the hardware only give 100 per second). Using images of patients involving different positions, the intelligent system is trained so that it was able to recognize different postures of patients on a simulator of the bed that was built (Fig. 5.2). The intelligent monitor helps to know if the patient is on a correct position in each different position of the bed. There are different positions that patients may have on the bed. These positions will be processed for analysis in different positions that the patient may have on the bed while this robot could be moving. These positions will be processed for real-time analysis and from that time the intelligent system can perform posture recognition right or wrong in a way that could indicate a total stoppage of the movement of the robotic bed. Fig. 5.2 show part of results of the data processing applied to the images obtained from the movements of the bed. After this step, then the artificial intelligent 


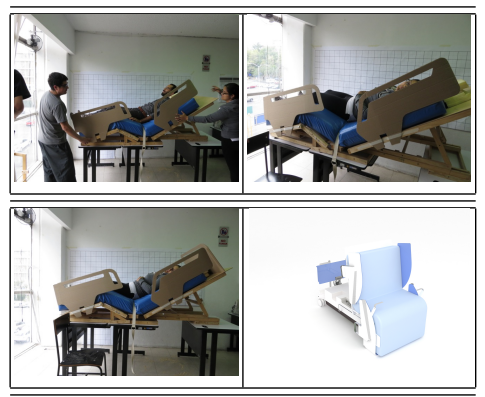

Fig. 9. Some positions of the robotic bed.

algorithm works in order to identify if the read position of patient is the right for the current position of the bed. Our team have developed a graphical interface

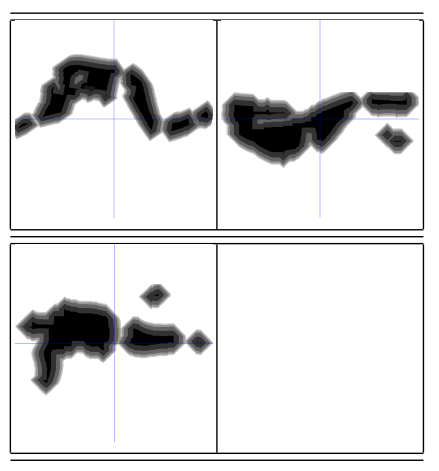

Fig. 10. Data pre-processing before the recognition step.

which help to visualize the results in terms of the identification and classification of the positions of the patient considering that robotic bed can be moving (Fig. $5.2)$.

\section{Conclusions}

Our device has a system which represents a strategy to endow robots assistants ability to detect risk scenarios to patients. In this case, when the robotic bed is moving can generate situations of risk if patients perform any bodily movement that is inappropriate to such a move. This represents an advance in medical care since through such devices can treat patients without medical personnel risk of an injury when moving patients personally. In this case, the robotic bed can be programed in such way the appropriate medical personnel may attend 


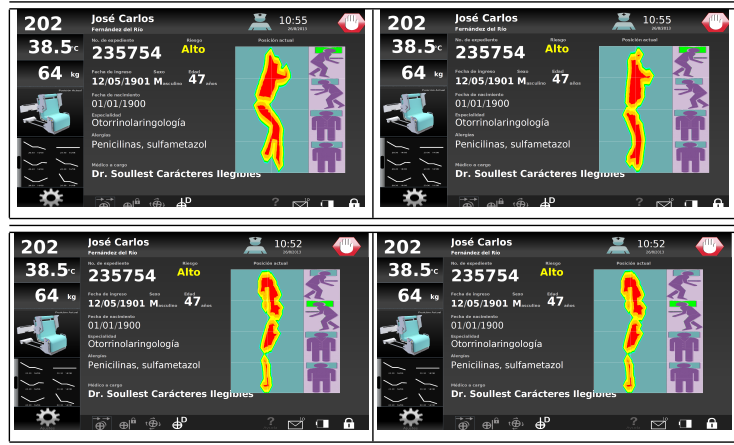

Fig. 11. A graphical interface. Patients positions recognized from patient movements data. The robotic bed could be moving.

to other patients while applying certain movement therapy automatically by medical monitoring. We have presented a methodology which is useful to classify the human bodies lying for real-time execution, the percentage of assertiveness is high, $99.01 \%$, this ensuring correct monitoring in the majority of cases, also we show the correct size of interpolated arrays to get the best results, so that the system is able to process the 100 arrays per second that hardware is able to deliver. These results allow us to observe that it is possible to identify in real time what the positions of the patient on the bed are. This allows us then command the robotic bed so that the mechatronic device can meet the intelligent monitor commands indicates. We are talking about the intelligent system would act as a robot controller and receives information from the environment. Today, we are currently developing the sub system to help us identify trends movement in order the robot bed reacts before the patient execute some movement. This will help prevent multiple scenarios of risk to patients. Likewise, we are working to develop strategies to ensure $100 \%$ detection of the positions of the patient to avoid the risk that is involved in the remaining $1 \%$ so far can not meet our development. It is important to emphasize that this intelligent device was designed and manufactured considering specific requirements indicated by medical personnel of Mexican public hospitals as previously explained. This is very important because the whole system responds to a social need and not stems from of an offer by the research experience. Our development is in the process of intellectual property protection, including all aspects of intelligent medical device. We believe that at present, the development of technology must focus on the specific demands of the market and society requires and not all should concentrate on proposals that scientific and research experience to offer. In this way, we would be connecting the strength of technology development with actual needs around us. 


\section{References}

1. Wang, Y., Butner, S. E., Darzi, A.: The Developing Market for Medical Robotics. Proceedings of the IEEE, Vol 94(9), pp. 1763-1771 (2006)

2. Ávila-Chaurand, R., Prado-León, L. R., Gonzlez-Muoz, E. L.: Dimensiones antropomtricas de población latinoamericana: Mxico, Cuba, Colombia, Chile. Universidad de Guadalajara, Centro Universitario de Arte, Arquitectura y Diseo, División de Tecnología y Procesos, Departamento de Producción y Desarrollo, Centro de Investigaciones en Ergonomá, 207 p. (2001)

3. Pavlovic, N. D., Milosevic, M., Pavlovic, N. T.: Development of Mechanisms for Adjusting Positions of a Multifunctional Bed. In: 13th World Congress in Mechanism and Machine Science, Guanajuato, Mxico, pp 1-6 (2011)

4. Mohammeda, M. N., Khrita, N. G., Abdelgneia, M. A., Abubakera, Es., Muftaha, A.F., Omara, M. Z., Salleha, M. S.: A New Design of Multi-Functional Portable Patient Bed. Jurnal Teknologi (Sciences \& Engineering), Vol. 58, pp. 61-66 (2012)

5. Ching-Hua, W., Ting-Chun, T., Shin-Chieh, H., Wan-Chun, C., Yen-Ming, C., Kun-Tse, T., Chun-Went, Y., Kuo-YiI, C.: Hospital Bed with Auxiliary Functions of Lateral Positioning and Transferring for im-iob ized atients. In: The 33rd Annual Conference of the IEEE Industrial Electronics Society (IECON), Taipei, Taiwan, pp. 2991-2995 (2007)

6. Shih-Wei P., Feng-Li L., Li-Chen F.: Mechanism Design and Mechatronic Control of a Multifunctional Test Bed for Bedridden Healthcare. IEEE/ASME Transactions on Mechatronics, Vol. 15(2), pp. 234-241 (2010)

7. Kittipichai, R., Ariyarit, A.: The Sizing Optimization of Hospital Bed Structure for Independently Supporting Left and or Right Leg Using Genetic Algorithms. International Journal of Modeling and Optimization, Vol. 11m no. 2, pp. 122-128 (2011)

8. Kap-Ho, S., Changmok, O., Tae-Yong, C., Ju-Jang, L.: Bed-type Robotic System for the Bedridden. In: Proceedings of the 2005 IEEE/ASME International Conference on Advanced Intelligent Mechatronics, Monterey, California, USA, pp. 1170-1175 (2005)

9. Andhare, A., Onkar, A., Padole, P.: Design of Bed for Bedridden Patients: Analysis and Synthesis of Mechanisms. In: 15th National Conference on Machines and Mechanisms, Chennai, Tamilnadu, India, pp. 1-6 (2011)

10. Vázquez-Santacruz, E., Gamboa-Zúñiga, M.: A diagnosis methodology for assistive technology development . In: Electrical Engineering, Computing Science and Automatic Control (CCE), 2013 10th International Conference on, pp. 163-169 (2013)

11. Beltrán-Herrera, A. Vázquez-Santacruz, E., Gamboa-Zúñiga. M.: Real-Time Classification of Lying Bodies by HOG Descriptors, In: Pattern Recognition 6th Mexican Conference, MCPR 2014, Cancun, Mexico, pp. 211-220 (2014)

12. Mehta, R. K., Horton, L. M., Agnew, M. J., Nussbaum, M. A.: Ergonomic evaluation of hospital bed design features during patient handling tasks. International Journal of Industrial Ergonomics, vol. 41, pp. 647-652 (2011)

13. Nicol, Klaus; Rusteberg, Dietmar: Pressure distribution on mattresses. Journal of Biomechanics, vol. 26, number 12, pp. 1479-1486 (1993)

14. James W. DeVocht, David G. Wilder, Eric R. Bandstra, Kevin F. Spratt: Biomechanical evaluation of four different mattresses. Applied Ergonomics, vol. 37, number 3, pp. 297-304 (2006) 
15. Townsend, D., Holtzman, Megan, Goubran, R., Frize, M., Knoefel, F.: Relative Thresholding With Under-Mattress Pressure Sensors to Detect Central Apnea. Instrumentation and Measurement, IEEE Transactions on, vol. 60, number 10, pp. 281-3289 (2011)

16. Amaya Arcelus, Christophe L. Herry, Rafik A. Goubran, Frank Knoefel, Heidi Sveistrup, Martin Bilodeau: Determination of Sit-to-Stand Transfer Duration Using Bed and Floor Pressure Sequences. IEEE Trans. Biomed. Engineering, vol. 56, number 10, pp. 2485-2492 (2009)

17. Dalal, Navneet; Triggs, Bill: Histograms of Oriented Gradients for Human Detection. In: Proceedings of the 2005 IEEE Computer Society Conference on Computer Vision and Pattern Recognition (CVPR'05), Volume 01, CVPR '05, pp. 886-893, IEEE Computer Society (2005)

18. Lowe, David G.: Object Recognition from Local Scale-Invariant Features. In: Proceedings of the International Conference on Computer Vision, Volume 2, ICCV'99, IEEE Computer Society (1999)

19. Robert Grimm; Sebastian Bauer: Johann Sukkau: Joachim Hornegger; Günther Greiner: Markerless estimation of patient orientation, posture and pose using range and pressure imaging. Int. J. Computer Assisted Radiology and Surgery, Volume 7, number 6, pp. 921-929 (2012)

20. Manuel Chica; Pascual Campoy; María A. Pérez; Tomás Rodríguez; Rubén Rodríguez; Óscar Valdemoros: Corrigendum to: Real-time recognition of patient intentions from sequences of pressure maps using artificial neural networks. Computers in Biology and Medicine, volume 43, number 9, pp. 1302 (2013)

21. Kap-Ho Seo; Tae-Yong Choi; Changmok Oh: Development of a robotic system for the bed-ridden. Mechatronics, volume 21, number 1, pp. 227-238 (2011)

22. Idzikowski, Christopher: Learn to Sleep Well. Watkins (2010)

23. Someya, Takao; Sekitani, Tsuyoshi; Iba, Shingo; Kato, Yusaku; Kawaguchi, Hiroshi; Sakurai, Takayasu: A large-area, flexible pressure sensor matrix with organic field-effect transistors for artificial skin applications. In: Proceedings of the National Academy of Sciences of the United States of America, volume 101, number 27, pp. 9966-9970 (2004)

24. Manunza I., Bonfiglio, A.: Pressure sensing using a completely flexible organic transistor. Biosensors and Bioelectronics, volume 22, number 12, pp. 2775-2779 (2007)

25. Mannsfeld, S. C. B., et. al.: Highly sensitive flexible pressure sensors with microstructured rubber dielectric layers. Nature Materials, volume 9, number 10, pp. 859-864 (2010)

26. Sekitani T., Zschieschang, U., Klauk, H., Someya, T.: Flexible organic transistors and circuits with extreme bending stability. Nature Materials, volume 9 , number 12, pp. $1015-1022$ (2010)

27. Sensing Tex Smart Textiles, Webpage: http://www.sensingtex.com/ (2013)

28. Byun, H., Lee, S.-W.: A survey on pattern recognition applications of support vector machines. International Journal of Pattern Recognition and Artificial Intelligence, volume 17, number 03, pp. 459-486 (2003)

29. Keerthi, S. Sathiya; Lin, Chih-Jen: Asymptotic Behaviors of Support Vector Machines with Gaussian Kernel. Neural Comput., July, volume 15, number 7, pp. 1667-1689, MIT Press (2003)

30. Cortes, Corinna and Vapnik, Vladimir: Support-Vector Networks. Mach. Learn., volume 20, number 3, pp. 273-297, Kluwer Academic Publishers (1995)

31. Ting, KaiMing: Confusion Matrix. In: Encyclopedia of Machine Learning, Sammut, Claude and Webb, Geoffrey (eds.), Springer US, pp. 209-209 (2010) 
32. Lowe, David G.: Distinctive Image Features from Scale-Invariant Keypoints. International Journal of Computer Vision. Kluwer Academic Publishers, pp. 91-110, volume 60, number 2 (2004)

33. Bay, Herbert; Ess, Andreas; Tuytelaars, Tinne; Van Gool, Luc,: Speeded-Up Robust Features (SURF). Comput. Vis. Image Underst., volume 110, number 3, pp. 346-359, Elsevier Science Inc. (2008)

34. Wang, Jian-Gang; Li, Jun; Lee, Chong Yee; Yau, Wei-Yun: Dense SIFT and Gabor descriptors-based face representation with applications to gender recognition. In: ICARCV, pp. 1860-1864, IEEE (2010)

35. Dalal, N. and Triggs, B.: Histograms of oriented gradients for human detection. In: Computer Vision and Pattern Recognition, CVPR 2005, IEEE Computer Society Conference on, volume 1, pp. 886-893 (2005)

36. , Hsu, C.-W., Chang, C.-C., and Lin, C.-J.: A practical guide to support vector classification. Tech. rep., Department of Computer Science, National Taiwan University (2003) 